\title{
Impact of regionalisation of a national rheumatic heart disease registry: the Ugandan experience
}

\author{
Emmy Okello, ${ }^{1,2}$ Chris T Longenecker, ${ }^{3,4}$ Amy Scheel, ${ }^{5}$ Twalib Aliku, ${ }^{6}$ \\ Joselyn Rwebembera, ${ }^{1,7}$ Grace Mirembe, ${ }^{8}$ Craig Sable, ${ }^{5}$ Peter Lwabi, ${ }^{1}$ Andrea Beaton ${ }^{5}$
}

\begin{abstract}
'Uganda Heart Institute, Kampala, Uganda

${ }^{2} \mathrm{~S}$ chool of Medicine, Makerere University, Kampala, Uganda

${ }^{3}$ Department of Medicine, Case Western Reserve University School of Medicine, Cleveland, Ohio, USA

${ }^{4}$ Division of Cardiology, University Hospitals of Cleveland, Cleveland, Ohio, USA ${ }^{5}$ Division of Cardiology, Children's National Health System, Washington DC, USA ${ }^{6}$ School of Medicine, Gulu University, Gulu, Uganda ${ }^{7}$ Mbarara University of Sciences and Technology, Mbarara, Uganda

${ }^{8}$ Joint Clinical Research Centre, Kampala, Uganda
\end{abstract}

\section{Correspondence to} Dr Andrea Beaton, Division of Cardiology, Children's National Health System, Washington DC 20010, USA; abeaton@ childrensnational.org

Received 16 November 2017 Revised 20 December 2017 Accepted 21 December 2017 Published Online First 13 January 2018
ABSTRACT

Objectives Rheumatic heart disease (RHD) remains a major driver of cardiovascular morbidity and mortality in low-resource settings. Registry-based care for RHD has been advocated as a powerful tool to improve clinical care and track quality metrics. Data collected through an RHD registry may also reveal epidemiological and geospatial trends, as well as insight into care utilisation. Uganda established a central RHD registry at the country's only tertiary cardiac centre in 2010. In 2014 RHD care and registry enrolment expanded to the Western region and in 2015 to the North. Here, we examine the geographical distribution of RHD cases in Uganda and the impact of registry expansion.

Methods A retrospective search of the Ugandan national RHD registry was preformed to capture all cases of acute rheumatic fever or clinical RHD from January 2010 through July 2016. A geospatial analysis revealed that the density of detected cases (cases/100 000 district residents) reflected proximity to an RHD registry enrolment centre. Regionalisation improved the number of cases detected in the regions of expansion and improved retention of patients in care.

Results and conclusions RHD appears to have uniform distribution throughout Uganda with geographical clustering surrounding RHD registry enrolment centres reflecting access to care, rather than differences in prevalence. Higher rates of case detection and improved retention in care with regionalisation highlight the urgent need for decentralisation of cardiovascular services. Future studies should examine sustainable models for cardiovascular care delivery, including task shifting of clinical care and echocardiography and use of telemedicine.

\section{INTRODUCTION}

Rheumatic heart disease (RHD) remains a leading cause of cardiovascular disability and death in low-income and middle-income countries, affecting at least 32.9 million persons. ${ }^{1}$ RHD is classically preceded by acute rheumatic fever (ARF), a systemic inflammatory response to untreated or undertreated group A streptococcal infection. While the optimal strategy for ARF/RHD control in low-income and middle-income countries remains under investigation, it is well established that secondary prophylaxis, or delivery of regular penicillin to prevent recurrent streptococcal infections, improves outcomes for patients following ARF or RHD diagnosis. ${ }^{2}$ Registry-based control programmes have been advocated as the ideal framework for delivering secondary prophylaxis and for establishing a national RHD control programme. ${ }^{4-6}$
Registries are also important for epidemiological surveillance: the quantification and localisation of RHD burden. These data are particularly useful for monitoring RHD trends, identifying high-risk populations and evaluating quality metrics. These data can then be translated into advocacy tools for prioritisation and expansion of RHD services. The Uganda RHD registry (the Registry) was established in 2010 at the country's only tertiary cardiac centre, the Uganda Heart Institute (UHI) in the capital city, Kampala. The Registry expanded in 2011 to include a second small enrolment site in Kampala (Lubowa, September, 2011). Local support and grant funding (Medtronic Foundation, RHD Action) allowed for transition of the Registry to an electronic web-based platform (REDCap) in 2013, and for dedicated central staff including registered nurses, data managers and physician supervisors. The Registry along with RHD centres of excellence expanded to regional referral hospitals in the West (Mbarara Regional Referral Hospital) in 2014 and to the North (Gulu Regional Referral Hospital) in 2015 , coupled with increased resources including expanded echocardiography, physician presence, skilled nursing and secondary prophylaxis clinics. Here, we use the Ugandan RHD registry to examine national case distribution and analyse how regionalisation of the Registry changed access to care.

\section{MATERIALS AND METHODS Ethics statement}

Prior to data analysis, all patient data were deidentified and anonymised to protect patient privacy and confidentiality.

\section{General design}

A retrospective search of the Ugandan national RHD registry was performed to capture all enrolled patients with clinical RHD from time of primary case enrolment (3 January 2010) until start of this analysis (1 July 2016).

\section{Data collection}

Registrants are organised according to centre of enrolment and categorised as ARF, clinical RHD (symptomatic or known ARF) or latent RHD (RHD detected through echocardiographic screening programmes). ${ }^{7-10}$ Cases of ARF were excluded, as they were inconsistently captured and few. Cases of latent RHD cases detected through echocardiographic screening programmes were excluded secondary to inherent geographical selection bias. UHI and the Lubowa site (only 28 registrants in this 
series) are within Kampala and service a demographically similar population, thus were included together.

Date of enrolment was recorded for all registrants and categorised by year and quarter (ie, 1.1=year 1 quarter 1 ). Demographic variables (gender and age at enrolment) and RHD severity category (mild, moderate, severe) ${ }^{6}$ were collected. Severity of RHD was classified according to the severity of the most affected valve. Primary address, as documented at enrolment, was recorded.

Place of residence in Uganda is classified into national administrative units with geographical areas defined from smallest to largest, as village, parish, subcounty, country, district, region and country. The primary address listed in the Registry was standardised against the most recent Ugandan census data (2014), and then translated into district (112 Ugandan districts) and region (Central, North, East and West) of residence (EO). If no primary address was recorded, or if the primary address was outside of Uganda, records were excluded from geospatial and other analyses.

\section{Statistical analysis}

A geospatial analysis was conducted to map and quantify the spatial heterogeneity of RHD in Uganda. Overall registry enrolment by district was mapped as a factor of number of cases per 100000 district residents according to the 2014 Uganda census district populations. Pre-expansion was defined as prior to regional expansion (3 January 2010 to 30 September 2015), and postexpansion was defined from the opening of the Registry in Mbarara until the end of this study (1 October 2015 to 1 July 2016). Percentage case enrolment by region was studied before and after regional expansion using a z-test of proportions (North, East, West and Central). Registrant age, gender, severity of RHD (severe disease, moderate disease, mild disease), ${ }^{6}$ and Euclidean distance (in kilometres) between the centroid of each district and the registrant RHD registry enrolment centre were compared before and after registry expansion using a t-test for continuous variables and $\chi^{2}$ or Fisher's exact test for categorical variables as appropriate. A subanalysis, limited to registrants enrolled after registry expansion but at least 12 months prior to data analysis, was conducted to examine if distance from enrolment centre $(\leq 50 \mathrm{~km}$ or $>50 \mathrm{~km})$ impacted retention in caredefined by at least one clinical follow-up visit within 12 months of enrolment. All statistical analyses were performed using MedCalc V.X (MedCalc Software, Ostend, Belgium). Results were considered significant at a level of $\mathrm{P} \leq 0.05$.

\section{RESULTS}

There were a total of 1471 patients enrolled in the national Ugandan RHD registry between January of 2010 and 1 August 2016. Of these 243 were excluded from this analysis: 12 for enrolment diagnosis of ARF and 231 for enrolment diagnosis of latent RHD (102 borderline RHD; 129 definite RHD). Of the remaining clinical RHD cases, 17 had missing address data that prohibited localisation of primary residence to a specific Ugandan district and 4 identified a primary residence in another country (3 Sudan, 1 Rwanda). These 21 cases were excluded from further analysis, leaving 1207 cases (figure 1).

Registry enrolment by year and quarter, and the total cumulative registered cases over time, is shown in figure 2. Enrolment was highest during the third quarter (July, August, September) for five of six complete calendar years (exception year 5), with overall total third quarter enrolments (July, August, September) being significantly higher than any other quarter $(\mathrm{P}<0.01)$. Basic

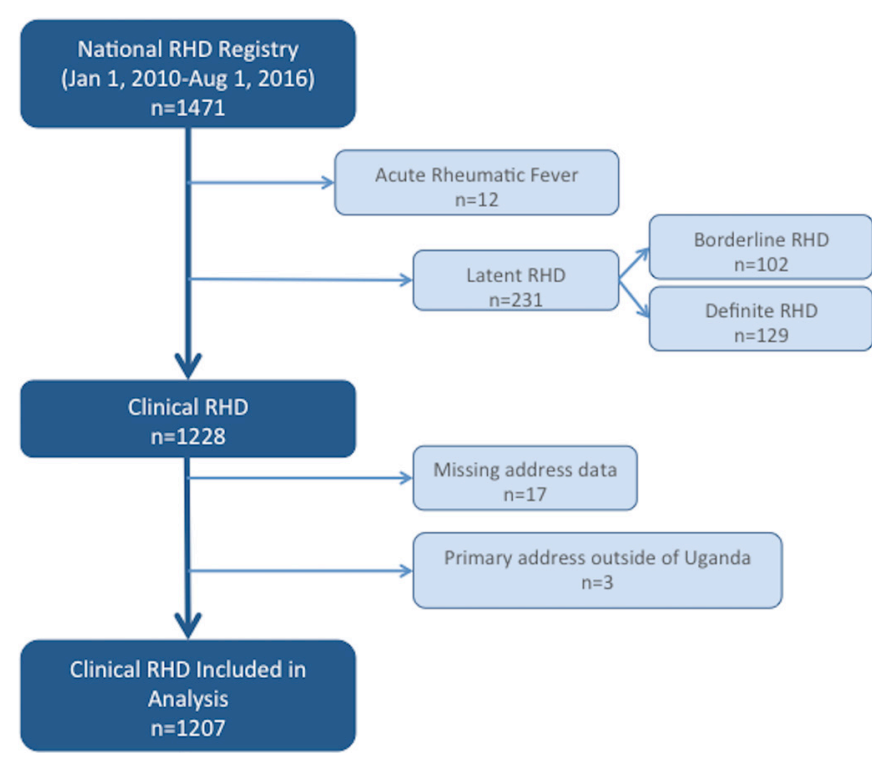

Figure 1 Selection of clinical rheumatic heart disease (RHD) cases for inclusion.

demographics of the registrants can be found in table 1 . Registrants were on average young $(24.2 \%<15 \%$ and $60.7 \%<30$ years), female $(70.8 \%)$ and with advanced RHD (73\% severe RHD). Registrants came from 80 of 112 districts (71\%): 18 of 24 districts in the Central region (75\%), 19 of 32 districts in the Eastern region (59\%), 23 of 30 districts in the Northern region (77\%), and 20 of 26 districts in the Western region (77\%).

Figure 3 shows a heat map of Ugandan districts, showing registry cases per 100000 residents. The catchment of regional RHD centres reflects their physical location, with many patients residing in the same district as the centre (63\% Gulu district, $38 \%$ Mbarara district) and almost all in the same region (98\% North/Gulu).

Of the 1207 included registrants, 845 were enrolled prior to regional registry expansion (average 63.5/month) and 362 after (45.3/month). Breakdown by enrolment centre shows 1100 cases at the main cardiology tertiary centre and a satellite enrolment centre in the capital (819 pre-expansion, 253 postexpansion), 63 cases at Gulu Regional Referral Hospital (North) and 44 cases at Mbarara Regional Referral Hospital (West). Registry expansion did not change registrant gender distribution or disease severity, but the age of registrants was slightly lowered postexpansion $(\mathrm{P}<0.01$, table 1$)$. Additionally, after regional expansion there

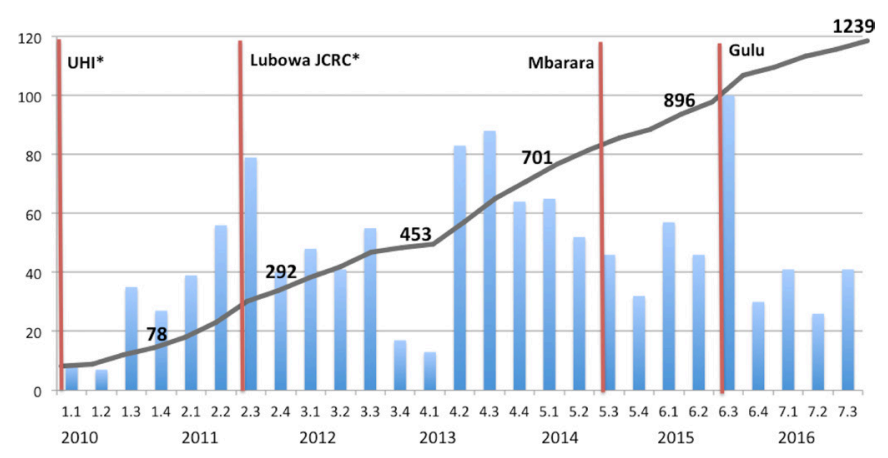

Figure 2 Registry enrolment by year and quarter. Red lines represent rheumatic heart disease (RHD) registry centre launch, while black trend line shows cumulative RHD cases enrolled over time. *Uganda Heart Institute (UHI) and Lubowa Joint Clinical Research Centre (JCRC) are considered together as 'Kampala' for these analyses. 
Table 1 Registrant demographics preregistry and postregistry expansion

\begin{tabular}{llll}
\hline & $\begin{array}{l}\text { Pre-expansion } \\
(\mathbf{n}=845)\end{array}$ & $\begin{array}{l}\text { Postexpansion } \\
(\mathbf{n}=362)\end{array}$ & P value \\
\hline $\begin{array}{l}\text { Enrolment age range, } \\
\text { mean } \pm \text { SD }\end{array}$ & $\begin{array}{l}3-78 \text { years } \\
28 \pm 15 \text { years }\end{array}$ & $\begin{array}{l}4-76 \text { years } \\
25 \pm 16 \text { years }\end{array}$ & $<0.01$ \\
Gender (\% female) & 71.5 & 69.3 & 0.43 \\
$\begin{array}{l}\text { Rheumatic heart disease } \\
\text { severity score }\end{array}$ & $\mathrm{n}=837$ & $\mathrm{n}=356$ & \\
$\begin{array}{l}\text { Mild (\%) } \\
\text { Moderate (\%) }\end{array}$ & 9 & 12.4 & 0.20 \\
Severe (\%) & 16.6 & 14.9 & \\
\hline
\end{tabular}

was significant change in proportional case enrolment by region of residence, with proportion of total registrants decreasing in the Central region and increasing in the Northern and Western regions $(\mathrm{P}<0.01$ for all). No change was seen in the Eastern region, where no regional expansion occurred (table 2). Similarly, the mean distance of the registrants from their primary address to the RHD registry enrolment site decreased for those coming from the North and West, but remained unchanged for those from the Central or Eastern regions (table 3).

The number of patients retained in clinical follow-up was also affected by distance between primary residence and RHD registry site. A subgroup analysis of 181 patients (136 Kampala, 21 Gulu and 24 Mbarara), enrolled between registry expansion and 1 August 2015 (to allow 12 months of follow-up), revealed only $36 \%$ of patients enrolled in Kampala had at least one clinical follow-up visit in the first year after enrolment. This was compared with $62 \%$ of those enrolled in Gulu $(\mathrm{P}<0.01)$ and $100 \%$ of those enrolled in Mbarara $(\mathrm{P}<0.01)$. Among those enrolled in Kampala, those who attended at least one follow-up visit were more likely to live $\leq 50 \mathrm{~km}$ from $\mathrm{UHI}(65.3 \%, \mathrm{P}=0.03)$.

\section{DISCUSSION}

RHD remains a major driver of cardiovascular morbidity and mortality in sub-Saharan Africa. Lack of robust epidemiological data and limited hospital and clinical records necessitate estimations of disease burden, which cannot provide granular data on patterns and distribution of disease. RHD registries, advocated as powerful tools for clinical care and epidemiological surveillance, can begin to fill this gap. Here, we use the national

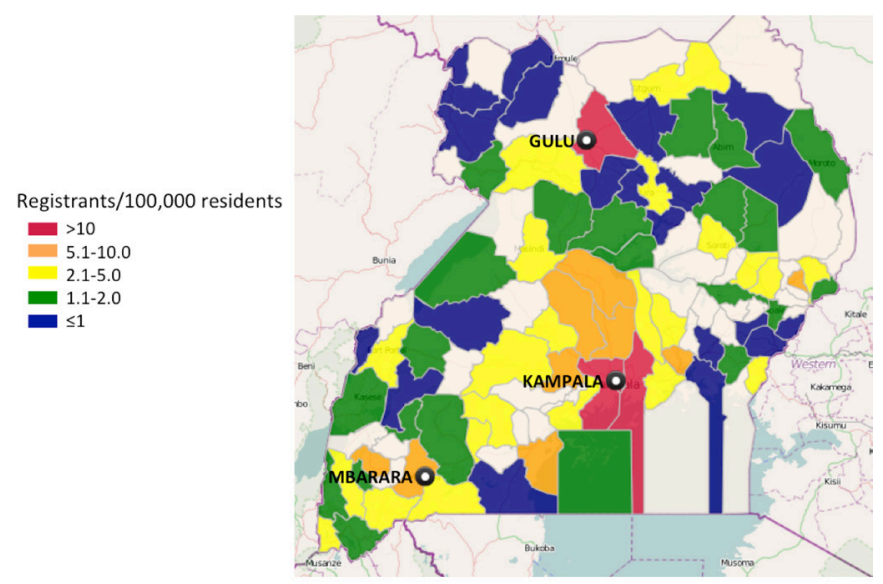

Figure 3 Rheumatic heart disease registry enrolment by district of residence (cases/100 000 district residents).
Table 2 Comparison of proportion of registrants by region of residence preregistry and postregistry expansion

\begin{tabular}{lllc}
\hline & Pre-expansion (\%) & Postexpansion (\%) & P value \\
\hline Central region & 70.2 & 48.1 & $<0.01$ \\
Eastern region & 12 & 11.6 & 0.73 \\
Northern region & 5.4 & 20.7 & $<0.01$ \\
Western region & 12.3 & 19.6 & $<0.01$ \\
\hline
\end{tabular}

RHD registry to analyse geospatial patterns of RHD distribution across Uganda preregistry and postregistry expansion. We uncovered that the distribution of RHD registrants reflects proximity to an enrolment centre, without other obvious clusters of high prevalence. Regionalisation of the Registry (to the North and West) increased the proportion of registrants coming from those regions and improved registrant retention in care.

Clustering of RHD cases around a centre of RHD care has been previously reported. A retrospective chart review of patients attending an adult cardiovascular clinic in Western Kenya showed over three-quarters of patients with RHD disease lived within $50 \mathrm{~km}$ of the study hospital. The authors postulated the effect could be secondary to selection bias-a combination of poor recognition of disease in more remote locations and lack of patient resources to travel to central tertiary centres. ${ }^{11}$ Regionalisation of the Ugandan RHD registry resulted in a significant increase in the proportion of RHD cases coming from the regions of expansion (North, West), with no increase in case detection in the Eastern region, where regionalisation did not occur. Clustering of cases was seen around all enrolment centres, suggesting the number of cases detected on the Registry reflected improved access to care. A community-based epidemiological RHD screening study is needed to truly estimate the national RHD burden and understand if there are higher and lower prevalence areas, but these data highlight the importance of decentralisation of cardiovascular services and establishment of at least regional centres for RHD diagnosis and care.

As part of these efforts, research should focus on the best roles for task shifting of RHD patient support and follow-up to nurses and other less-specialised providers. Task shifting has shown promise for developing an RHD screening workforce, ${ }^{10}{ }^{12-14}$ and may also be a successful strategy for expanding basic RHD clinical care, running secondary prophylaxis clinics, and leading patient and community engagement activities that could improve awareness and increase retention in care.

In addition to increased case detection, regionalisation of the Registry also improved retention in care, by decreasing the geographical distance between patients and providers. The impact of skilled nursing, patient outreach to improve retention and dedicated secondary prophylaxis clinics cannot be easily quantified, but likely played a critical role in this achievement. Retention in care is critical as patients with advanced RHD (73\%

Table 3 Comparison of mean distance between primary address and rheumatic heart disease registry enrolment site preregistry and postregistry expansion

\begin{tabular}{lccc}
\hline & Pre-expansion $\left(\mathrm{km}^{*}\right)$ & Postexpansion $\left(\mathrm{km}^{*}\right)$ & P value \\
\hline Central region & $25.4( \pm 36.0)$ & $24.1( \pm 48.8)$ & 0.63 \\
Eastern region & $135( \pm 59.9)$ & $135.7( \pm 68.3)$ & 0.49 \\
Northern region & $273( \pm 71.8)$ & $75.4( \pm 108.0)$ & $<0.01$ \\
Western region & $270.4( \pm 55.8)$ & $137.0( \pm 122.3)$ & $<0.01$ \\
\hline
\end{tabular}

* Mean Euclidean distance and SD. 
of this cohort) typically require multiple medications, are at high risk of cardiovascular complications and require regular evaluation of cardiac rhythm. Further, while this analysis did not directly assess adherence to secondary prophylaxis, patients in Uganda who are retained in care are more likely to be adherent, a factor that is critically linked to improved outcomes. ${ }^{15-17}$ Future research should include utilisation of the RHD registry to deliver innovative programmes that improve adherence and tracking of secondary prophylaxis.

While not the main focus of this study, the basic demographics of the Registry mirror other reports from Africa. As was seen in the REMEDY study, a multicentre, international hospital-based prospective RHD registry, the majority of patients on the Ugandan RHD registry were female (70\% in our study, $66.2 \%$ in REMEDY) and young (mean age 26 years in our study, 28 years in REMEDY). ${ }^{18}$ Regionalisation of RHD registry enrolment slightly but significantly lowered the mean age of registrants, suggesting decentralisation may improve access to diagnosis for vulnerable paediatric populations. It is also possible that RHD school screening programmes (which occurred alongside registry expansion in Gulu and Mbarara) increased case presentation. While cases of latent RHD detected through school screening were excluded from this analysis secondary to inherent geographical selection bias, it is possible that these programmes increased overall awareness of RHD among children and families, thus increasing presentation of clinical RHD.

There was no difference in the severity of registrant heart disease preregistry and postregistry expansion. Overwhelmingly, patients presenting for diagnosis and registry enrolment had severe disease (73\%), a finding that was also seen in Kenya, ${ }^{11}$ and in West and Central Africa. ${ }^{19}$ The ratio of severe RHD to any RHD at diagnosis negatively correlates with gross domestic product, ${ }^{19}$ potentially implicating lack of access to healthcare and poor health-seeking behaviour.

Early diagnosis is also impaired by the striking absence of ARF in sub-Saharan Africa. ${ }^{15} 20$ On the Ugandan RHD registry, only 12 patients over 6 years were enrolled with primary ARF. Lack of disease recognition in the community, presentation to lower levels of the health system and inconsistent enrolment of cases at the Registry centres could be contributors to low case detection. Recent modification of the Jones Criteria may improve the sensitivity of ARF case detection in high-risk populations, ${ }^{21}$ but epidemiological research is also needed to understand the gap between ARF and RHD burden.

An unexpected and consistent difference in enrolment by quarter was seen with five of six calendar years, having peak enrolment during the third quarter (July, August, September). While this finding is only hypothesis-generating, it may be of interest for health services planning. Uganda is an equatorial nation with little year-round fluctuation in temperature, although there are wet seasons, mid-September to November and from March to May in the South and March to November in the North. The third quarter falls in the dry season for much of the country (most of the Central, Western and Eastern regions), and increased enrolment may reflect better road conditions and easier transportation to RHD enrolment centres and/or a less demanding agricultural season, freeing farmers (majority of those living in rural areas) to travel and seek care.

\section{Limitations}

Our study has several limitations that should be considered when interpreting the results. This was a retrospective study, and classification of RHD, echocardiographic findings and clinical findings (which impact severity of disease classification) were

\section{Key messages}

\section{What is already known about this subject?}

Rheumatic heart disease (RHD) remains the most common acquired cardiovascular disease among children and young adults living in low-resource settings, with a high burden of disease in sub-Saharan Africa. Registry-based care has been advocated as the best way to care for these patients, providing a platform for case tracking and epidemiological surveillance.

\section{What does this study add?}

Analysis of geospatial data from registries may identify clusters of high prevalence areas or may reflect issues related to access to care. Study of the Ugandan RHD registry expansion revealed that regionalisation improved case detection in the region where the expansion occurred and improved retention in care.

\section{How might this impact on clinical practice?} Our study highlights the urgent need for decentralisation of cardiovascular services in RHD endemic areas. Future studies should examine sustainable models for cardiovascular care delivery, including task shifting of clinical care and echocardiography and use of telemedicine.

not independently verified. However, expert cardiologists or other RHD trained staff completed the final RHD diagnosis and echocardiographic review at enrolment, and echocardiograms were performed on all registrants to confirm RHD. Additionally, our convenience sampling and limited enrolment time at each expansion site resulted in small numbers in many of our analyses, which could have provided insufficient power to detect all impacts. However, in most cases the effects seen with regionalisation were large and $\mathrm{P}$ values highly significant.

\section{CONCLUSIONS}

In conclusion, RHD registry enrolment shows strong geographical clustering surrounding RHD registry enrolment centres reflecting access to care, rather than differences in prevalence. Higher rates of case detection and improved retention in care with regionalisation highlight the urgent need for decentralisation of cardiovascular services. Future studies should examine sustainable models for cardiovascular care delivery, including task shifting of clinical care and echocardiography and use of telemedicine.

Acknowledgements We would like to thank the Joint Clinical Research Centre staff members and clinical staff who support the Uganda national RHD registry in Lubowa, Mbarara and Gulu. In addition, a special thanks to the staff, administrators and hospital leadership at the Uganda Heart Institute for their support and commitment to reducing the burden of RHD in Uganda.

Contributors EO planned the study, analysed data and coauthored the first manuscript draft. CTL, AS, TA, JR, GM, CS and PL planned the study and critically reviewed the manuscript for scientific content. $A B$ planned the study, analysed the data and coauthored the first manuscript draft.

Funding This work was supported by the Children's National Global Health Initiative. Further support came from the Medtronic Global Health Foundation, Gift of Life International and General Electric.

\section{Competing interests None declared.}

Ethics approval Approval for this study was granted by the institutional review boards at Children's National Health System, Washington DC (Pro00000451), Case Western Reserve University, Cleveland, Ohio (03-13-42), Makerere University College of Health Science, Kampala, Uganda (REF 2013-072) and the Uganda National Council for Science and Technology.

Provenance and peer review Not commissioned; externally peer reviewed. 
Data sharing statement All deidentified study data are available for use on request to the corresponding author.

(c) Article author(s) (or their employer(s) unless otherwise stated in the text of the article) 2018. All rights reserved. No commercial use is permitted unless otherwise expressly granted.

\section{REFERENCES}

1 Global Burden of Disease Study 2013 Collaborators. Global, regional, and national incidence, prevalence, and years lived with disability for 301 acute and chronic diseases and injuries in 188 countries, 1990-2013: a systematic analysis for the Global Burden of Disease Study 2013. Lancet 2015;386.

2 Feinstein AR, Wood HF, Spagnuolo M, et al. Rheumatic fever in children and adolescents. A long-term epidemiologic study of subsequent prophylaxis, streptococcal infections, and clinical sequelae. Vii. Cardiac changes and sequelae. Ann Intern Med 1964;60:87-123.

3 Tompkins DG, Boxerbaum B, Liebman J. Long-term prognosis of rheumatic fever patients receiving regular intramuscular benzathine penicillin. Circulation 1972;45:543-51.

4 Rheumatic fever and rheumatic heart disease. World Health Organ Tech Rep Ser 2004;923:1-122.

5 Remenyi B, Carapetis J, Wyber R, et al. Position statement of the world heart federation on the prevention and control of rheumatic heart disease. Nat Rev Cardiol 2013;10:284-92.

6 McDonald M, Brown A, Noonan S, et al. Preventing recurrent rheumatic fever: the role of register based programmes. Heart 2005;91:1131-3.

7 Reményi B, Wilson N, Steer A, et al. World heart federation criteria for echocardiographic diagnosis of rheumatic heart disease--an evidence-based guideline. Nat Rev Cardiol 2012;9:297-309.

8 Beaton A, Okello E, Lwabi P, et al. Echocardiography screening for rheumatic heart disease in Ugandan schoolchildren. Circulation 2012;125:3127-32.

9 Beaton A, Lu JC, Aliku T, et al. The utility of handheld echocardiography for early rheumatic heart disease diagnosis: a field study. Eur Heart I Cardiovasc Imaging 2015;16:475-82.
10 Ploutz M, Lu JC, Scheel J, et al. Handheld echocardiographic screening for rheumatic heart disease by non-experts. Heart 2016;102:35-9.

11 Lumsden RH, Akwanalo C, Chepkwony S, et al. Clinical and geographic patterns of rheumatic heart disease in outpatients attending cardiology clinic in western Kenya. Int J Cardiol 2016;223:228-35.

12 Mirabel M, Bacquelin R, Tafflet M, et al. Screening for rheumatic heart disease: evaluation of a focused cardiac ultrasound approach. Circ Cardiovasc Imaging 2015;8:e002324.

13 Engelman D, Kado JH, Reményi B, et al. Screening for rheumatic heart disease: quality and agreement of focused cardiac ultrasound by briefly trained health workers. BMC Cardiovasc Disord 2016;16:30.

14 Beaton A, Nascimento BR, Diamantino AC, et al. Efficacy of a standardized computerbased training curriculum to teach echocardiographic identification of rheumatic heart disease to nonexpert users. Am J Cardiol 2016;117:1783-9.

15 Okello E, Wanzhu Z, Musoke C, et al. Cardiovascular complications in newly diagnosed rheumatic heart disease patients at Mulago Hospital, Uganda. Cardiovasc J Afr 2013;24:76-9.

16 Huck DM, Nalubwama H, Longenecker CT, et al. A qualitative examination of secondary prophylaxis in rheumatic heart disease: factors influencing adherence to secondary prophylaxis in Uganda. Glob Heart 2015;10:63-9.

17 Musoke C, Mondo CK, Okello E, et al. Benzathine penicillin adherence for secondary prophylaxis among patients affected with rheumatic heart disease attending Mulago Hospital. Cardiovasc J Afr 2013;24:124-9.

18 Zühlke L, Engel ME, Karthikeyan G, et al. Characteristics, complications, and gaps in evidence-based interventions in rheumatic heart disease: the Global Rheumatic Heart Disease Registry (the REMEDY study). Eur Heart J 2015;36:1115-22.

19 Kingué S, Ba SA, Balde D, et al. The VALVAFRIC study: a registry of rheumatic heart disease in Western and Central Africa. Arch Cardiovasc Dis 2016;109:321-9.

20 Zhang W, Mondo C, Okello E, et al. Presenting features of newly diagnosed rheumatic heart disease patients in Mulago Hospital: a pilot study. Cardiovasc J Afr 2013;24:28-33.

21 Gewitz MH, Baltimore RS, Tani LY, et al. Revision of the Jones Criteria for the diagnosis of acute rheumatic fever in the era of Doppler echocardiography: a scientific statement from the American Heart Association. Circulation 2015;131:1806-18. 